\title{
Structural Reliability Analysis via the Multivariate Gegenbauer Polynomial-Based Sparse Surrogate Model
}

\author{
Yixuan Dong and Shijie Wang \\ School of Mechanical Engineering, Shenyang University of Technology, Shenyang 110870, Liaoning, China \\ Correspondence should be addressed to Shijie Wang; sjwang_syut@163.com
}

Received 8 September 2021; Revised 1 October 2021; Accepted 8 October 2021; Published 27 October 2021

Academic Editor: Debiao Meng

Copyright (C) 2021 Yixuan Dong and Shijie Wang. This is an open access article distributed under the Creative Commons Attribution License, which permits unrestricted use, distribution, and reproduction in any medium, provided the original work is properly cited.

\begin{abstract}
Structural reliability analysis is usually realized based on a multivariate performance function that depicts failure mechanisms of a structural system. The intensively computational cost of the brutal-force Monte-Carlo simulation motivates proposing a Gegenbauer polynomial-based surrogate model for effective structural reliability analysis in this paper. By utilizing the orthogonal matching pursuit algorithm to detect significant explanatory variables at first, a small number of samples are used to determine a reliable approximation result of the structural performance function. Several numerical examples in the literature are presented to demonstrate potential applications of the Gegenbauer polynomial-based sparse surrogate model. Accurate results have justified the effectiveness of the proposed approach in dealing with various structural reliability problems.
\end{abstract}

\section{Introduction}

Structural reliability analysis needs to recursively run a performance function $g(\mathbf{X})$ for an accurate estimation result of the structural failure probability. Herein, the vector $\mathbf{X}=$ $\left[X_{1}, \ldots, X_{d}\right]^{T}$ comprises all input random variables, and each pair of $X_{i}$ and $X_{j}$ are assumed as independent for the sake of simplicity. Or else, statistical transformations in the literature are required to obtain equivalently independent random variables [1-3]. Note that the performance function is highly possible to be implicitly expressed and computationally expensive, which makes the corresponding structural reliability analysis be not an easy but highly demanding task in reality.

Analytical derivation for stochastic characteristics of the multivariate performance function is only feasible in rare cases. The Monte Carlo simulation (MCS) and its variants allow one to alleviate the difficulty to some extent [4-6]. However, a simulation-based approach might become tedious due to high computational efforts [7]. Approximation methods, for example, the first-order reliability method (FORM) were widely investigated [8]. This paper primarily focuses on the utility of the Gegenbauer polynomials for a sparse surrogate model to mimic the true but computationally demanding performance function in structural reliability analysis. To achieve this, the orthogonal matching pursuit (OMP) in the signal processing domain is introduced to determine the principle explanatory polynomials for a sparse model, and the chaotic Gegenbauer polynomials are Directional MCS00 introduced in this paper to realize the sparse regression, rather than ordinary polynomials $\left\{1, x, x^{2}, \ldots\right\}$ in conventional response surface methods (RSMs).

In reality, the RSM has received considerable attention over the past decades [9-11]. A general procedure to build the surrogate model usually comprises two aspects: (1) to locate feasible combinations of explanatory variables and their coefficients for the optimized mean response and (2) to estimate the model response in the vicinity of the optimized location for better understanding the of the "local" effect of factors [12]. Once a group of explanatory variables are available, the least-square regression and the error backprorogation mechanism are combined to determine unknown parameters [13-16].

Consider that all explanatory variables need to be fully assessed in the conventional regression analysis. The total 
number of model terms will be exponentially increased with the dimensionality of input variables. The curse of dimensionality makes the conventional RSM be usually applicable to low-dimensional problems. Instead, the sparse regression analysis aims to use a fairly small number of regressors to overcome the disadvantage. Combined with the Galerkin projection [17] or the stochastic collocation method [18] to calculate the coefficients, the determined surrogate models have been widely used for uncertainty quantification of various structural systems [19-21].

The paper proposes to build a sparse surrogate model for structural reliability analysis via the Gegenbauer polynomials. The univariate Gegenbauer polynomials $G_{k}^{(\beta)}(x)$ (as $k=0,1, \ldots)$ are specified by the weight function $w(x)=$ $\left(1-x^{2}\right)^{\beta-1 / 2}$ and are constituted as a complete basis to represent an arbitrary function defined on $\forall x \in[-1,1][22]$. Given various realizations of the polynomial parameter $\beta$, $G_{k}^{(\beta)}(x)$ define a group of polynomials for the model approximation. For instance, $\beta=1 / 2$ defines the Legendre polynomial, whereas $\beta=0$ and $\beta=1$ are related to the first and the second kinds of the Chebyshev polynomials. Flexility motivates to utilize the Gegenbauer polynomials to develop surrogate models for the structural reliability analysis in this paper.

Following the theorem of the generalized Furious expansion, a real-valued function can be exactly represented via a complete basis set. Results from the variance-based global sensitivity analysis have vividly demonstrated utilizing a small number of principle terms for an accurate estimation of the true model. This motivates developing a sparse approximation of the multivariate performance function. Once the multivariate basis set based on the Gegenbauer chaos polynomials is constituted, the method of the orthogonal matching pursuit is further introduced to select primary functions to maintain the high sparsity of the surrogate model. Similar algorithms include the basis pursuit [23] and the matching pursuit methods [24], or the method via the numerical approach of the orthogonal matching pursuit (OMP) [25].

The OMP algorithm has been widely recognized to select principle regressors that are correlated with residual errors of an engineering model. Relying on the orthogonal projection of residual errors to the linear space spanned by orthogonal polynomials, the OMP algorithm can iteratively minimize the global model error sparsely [26]. Due to its advantages in terms of simple implementation and fast convergence, the OMP has been successively used for data compression, signal recovery, image recognition, and so on. Besides, advanced algorithms such as the stagewise orthogonal matching pursuit (StOMP) and the regularized orthogonal matching pursuit (ROMP) were developed in $[27,28]$. The paper introduces the Gegenbauer polynomials to constitute the basis functions, and a variety of stopping criteria are further investigated for a robust OMP-based sparse regression model in structural reliability analysis. The approach is nonintrusive, which implies the corresponding structural reliability analysis is only based on a small number of deterministic model evaluations, rather than requiring high order responses, for example, the gradients in the firstorder reliability method (FORM) [29].

An objective of the paper is to utilize the multivariate Gegenbauer polynomials as the basis function for an effective surrogate model for the structural reliability analysis. The high-dimensional Gegenbauer polynomials are first presented to define the multivariate chaos terms. Combined with a variety of stopping criteria to realize the sparse regression, an effective surrogate model that mimics the true but computationally demanding performance function is determined for the structural reliability analysis.

The rest of the manuscript is organized as follows: Section 2 briefly summarizes the mathematic properties of the Gegenbauer polynomials, and the multivariate basis functions are further formulated by the chaotic multiplication of univariate functions. Section 3 presents several stopping criteria for a robust sparse regression result. $\mathrm{Nu}-$ merical examples in Section 4 are presented to demonstrate potential applications of the proposed sparse regression model, and conclusions are summarized in Section 5.

\section{The multivariate Gegenbauer Polynomials}

A reliable sparse regression result for a structural performance function depends largely on the multivariate basis functions to span the approximation space. The paper presents utilizing the chaotic multivariate Gegenbauer polynomials. Therefore, the section starts to introduce mathematical characteristics of the univariate Gegenbauer polynomials, and multivariate basis functions are further derived via the chaotic multiplication of one-dimensional polynomials.

2.1. The Gegenbauer Polynomial. The Gegenbauer polynomials have been widely treated as a group of basis functions for the numerical approximation in engineering realities [30]. In mathematics, $G_{k}^{(\beta)}(x)(k=0,1, \ldots)$ are defined as pairwise orthogonal polynomials for $x \in[-1,1]$. They are particular solutions of the Gegenbauer differential equation [31]:

$$
\left(1-x^{2}\right) y^{\prime \prime}-(2 \beta+1) \mathrm{xy}^{\prime}+k(k+2 \beta) y=0,
$$

which can be further degenerated as the Legendre and Chebyshev differential equation, and the Legendre and the Chebyshev polynomials are two special cases of the Gegenbauer polynomials as shown in numerical simulations.

Besides, the Gegenbauer polynomial $G_{k}^{(\beta)}(x)$ can be expressed via the generating function as well [32]:

$$
\frac{1}{\left(1-2 \mathrm{xt}+t^{2}\right)^{\beta}}=\sum_{k=0}^{\infty} G_{k}^{(\beta)}(x) t^{k},
$$

and the recurrence relation of the orthogonal polynomials is given as 


$$
\left\{\begin{array}{l}
G_{0}^{(\beta)}(x)=1, \\
G_{1}^{(\beta)}(x)=2 \beta x, \\
k G_{k}^{(\beta)}(x)=2 x(k+\beta-1) G_{k-1}^{(\beta)}(x)-(k+2 \beta-2) G_{k-2}^{(\beta)}(x) \quad(k \geq 2) .
\end{array}\right.
$$
by

Mathematically, the orthogonality of $G_{k}^{(\beta)}(x)$ is governed

$$
\int_{x} w(x) G_{k}^{(\beta)}(x) G_{l}^{(\beta)}(x) \mathrm{d} x=C_{k}(\beta) \delta_{k l} .
$$

Herein, $w(x)=\left(1-x^{2}\right)^{\beta-1 / 2}$ denotes the weight function $x \in[-1,1]$, and $\delta_{m n}$ represents the Kronecker delta symbol. This is further used to define the normalization constant $C_{k}(\beta)$ :

$$
C_{k}(\beta)=\frac{\pi 2^{1-2 \beta} \Gamma(k+2 \beta)}{k !(k+\beta)[\Gamma(\beta)]^{2}} \quad(k=0,1, \ldots),
$$

in which the symbol $\Gamma(\cdot)$ denotes the Gamma function.

Given the parameter $\beta=1 / 2,1.0,2.0$, and 3.0, the first six realizations of the Gegenbauer polynomial (i.e. $k=0, \ldots, 5$ ) are presented in Figure 1. The Legendre and the Chebyshev polynomials are two special cases of the Gegenbauer polynomial, which will be utilized to build multivariate basis functions for a sparse approximation result of the performance function $g(\mathbf{X})$ in structural reliability analysis.

2.2. The multivariate Gegenbauer polynomial. Structural reliability analysis is always evaluated based on a multivariate performance function $g(\mathbf{X})$, and the multivariate Gegenbauer polynomials will be derived for a numerical approximation of the performance function.
Define an index vector $\theta=\left[\theta_{1}, \ldots, \theta_{d}\right]^{T}$ with each integral $\theta_{k} \in[0, p]$. Then, the degree of a $d$-variate chaotic Gegenbauer polynomial $\phi_{\theta}(\mathbf{x})$ can be measured by the length of the index vector, that is, $|\theta|=\sum_{k=1}^{d} \theta_{k}$. Specifically, the total number $(N)$ of chaotic terms in the polynomial set $\left\{\phi_{\theta}(\mathbf{x})\right\}$ is determined by parameters $d$ and the polynomial order $p$ as

$$
N=\left(\begin{array}{c}
d+p \\
p
\end{array}\right)=\frac{(d+p) !}{d ! p !},
$$

and the vector-indexed multivariate Gegenbauer polynomials are defined via the chaotic multiplication of the univariate functions:

$$
\left\{\phi_{i}(x), i=0, \ldots, N-1\right\}:=\underset{|\theta| \leq \prod_{k=1}^{U}}{U} G_{\theta_{k}}^{(\beta)}\left(x_{k}\right),
$$

wherein $G_{\theta_{k}}^{(\beta)}\left(x_{k}\right)$ represents the $\theta_{k}$ th-order univariate polynomial presented in Section 2.1.

Figure 2 presents the bivariate Gegenbauer polynomials with parameters $\beta=1 / 2$ and the polynomial degree $p=4$. For other high-dimensional cases, one can refer to the tensor-product formulation in equation (7). Note that each two pair of multivariate Gegenbauer polynomials are orthogonally defined, given that

$$
\left\langle\phi_{i}(\mathbf{x}), \phi_{j}(\mathbf{x})\right\rangle=\int_{\mathbf{x}} W(\mathbf{x}) \phi_{i}(\mathbf{x}) \phi_{j}(\mathbf{x}) \mathrm{d} \mathbf{x}=C_{0} \delta_{i j} \quad(i, j=0, \ldots, N-1),
$$

wherein $W(\mathbf{x})$ represents a $d$-dimensional weighting function $W(\mathbf{x})=\prod_{k=1}^{d}\left(1-x_{k}^{2}\right)^{\beta-1 / 2}$ as $\mathbf{x} \in[-1,1]^{d}$ whereas $C_{0}$ denotes a normalization constant.

To illustrate the orthogonal characteristic, the original index vector of an arbitrary two orders of the chaotic polynomials are recovered as $\theta^{(i)}=\left[\theta_{1}^{(i)}, \theta_{2}^{(i)}, \ldots, \theta_{d}^{(i)}\right]^{T}$ and $\theta^{(j)}=\left[\theta_{1}^{(j)}, \theta_{2}^{(j)}, \ldots, \theta_{d}^{(j)}\right]^{T}$, respectively. Following this notation, the inner product in equation (8) is realized as

$$
\begin{aligned}
\left\langle\phi_{i}(\mathbf{x}), \phi_{j}(\mathbf{x})\right\rangle & =\int_{\Omega} W(\mathbf{x}) \phi_{\boldsymbol{\theta}^{(i)}}(\mathbf{x}) \phi_{\boldsymbol{\theta}^{(j)}}(\mathbf{x}) \mathrm{d} \mathbf{x} \\
& =\prod_{k=1}^{d} \int_{x_{k}} w\left(x_{k}\right) G_{\theta_{k}^{(i)}}^{(\beta)}\left(x_{k}\right) G_{\theta_{k}^{(j)}}^{(\beta)}\left(x_{k}\right) \mathrm{d} x_{k}=\prod_{k=1}^{d} C_{\theta_{k}^{(i)}}(\beta) \delta_{\theta_{k}^{(i)} \theta_{k}^{(j)}}
\end{aligned}
$$

Note that $\forall \theta_{k}^{(i)} \neq \theta_{k}^{(j)}$ and $\phi_{i}(\mathbf{x}) \neq \phi_{j}(\mathbf{x})$ as $k=1, \ldots, d$ and $i, j=0, \ldots, N-1$. $\widehat{G}_{\theta_{k}}^{(\beta)}(x)=\frac{1}{\sqrt{C_{\theta_{k}}(\beta)}} \cdot G_{\theta_{k}}^{(\beta)}(x) \quad\left(\right.$ for each integer $\left.\theta_{k} \in[0, p]\right)$

Define the normalized univariate Gegenbauer polynomial: 


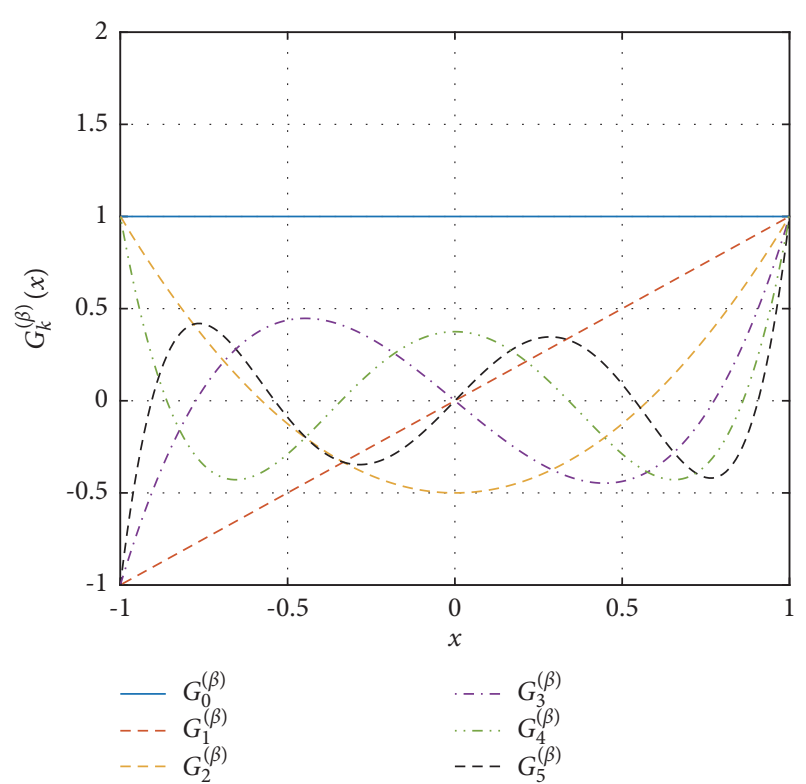

(a)

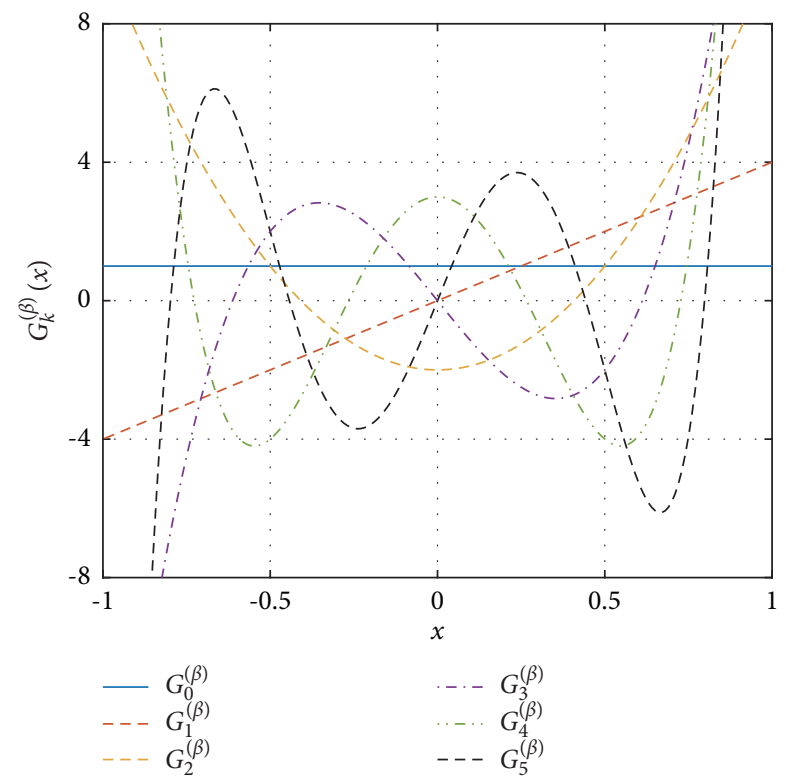

(c)

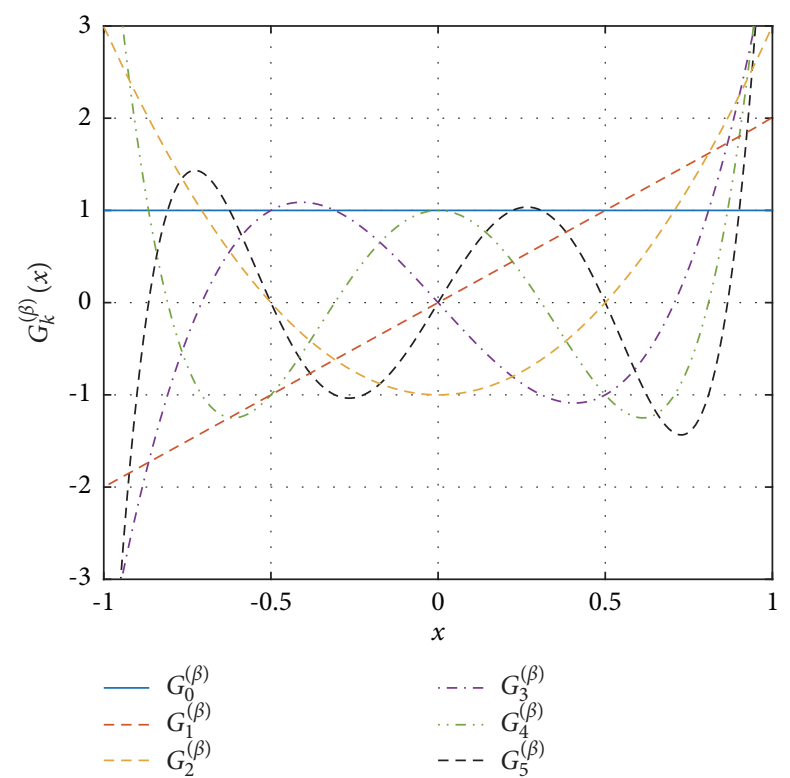

(b)

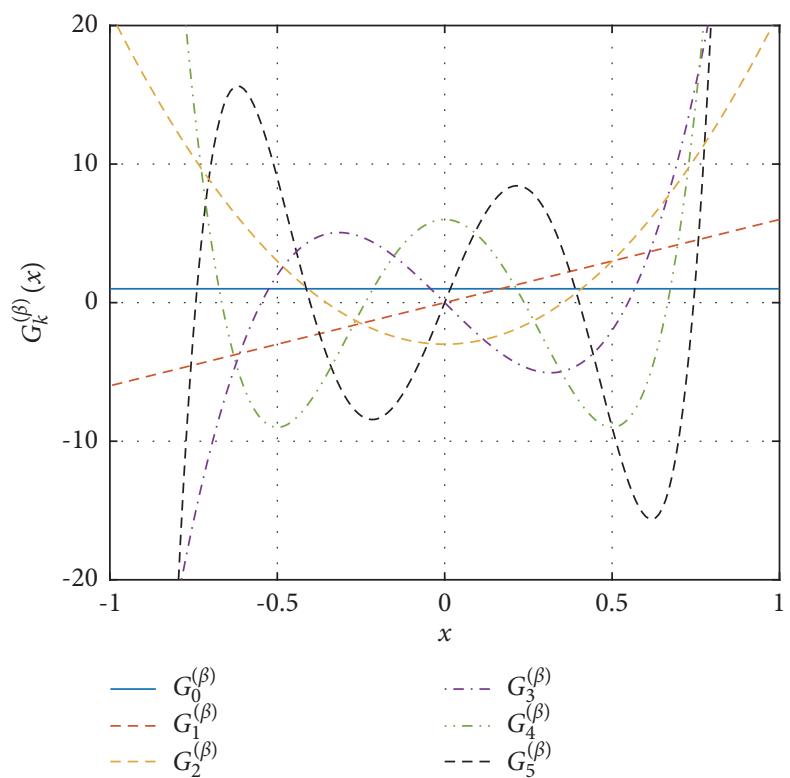

(d)

FiguRE 1: The first six orders of the Gegenbauer polynomial with various realizations of the parameter $\beta=1 / 2,1.0,2.0$, and 3.0. (a) $\beta=1 / 2$ : the Legendre polynomial. (b) $\beta=1$ : the Chebyshev polynomial. (c) $\beta=2.0$. (d) $\beta=3.0$.

Substituting $\widehat{G}_{\theta_{k}}^{(\beta)}(x)$ to equation $(8)$, one can obtain the normalized multivariate set $\left\{\widehat{\phi}_{0}(\mathbf{x}), \ldots, \widehat{\phi}_{N-1}(\mathbf{x})\right\}$, whose elements have the property of

$$
\left\langle\widehat{\phi}_{i}(\mathbf{x}), \widehat{\phi}_{j}(\mathbf{x})\right\rangle=\delta_{i j} \quad(i, j=0, \ldots, N-1),
$$

and its second-norm can be defined as

$$
\left\|\widehat{\phi}_{i}(\mathbf{x})\right\|_{2}=1 \quad(i=0, \ldots, N-1) .
$$

Note that the normalized multivariate Gegenbauer polynomials $\widehat{\phi}_{i}(\mathbf{x})$ (for $i=0, \ldots, N-1$ ) will be used as the basis functions in subsequent sparse approximations, and the normalized polynomial will be denoted as $\phi_{i}(\mathbf{x})$ for the sake of simplicity.

Besides the normalization issue, a linear transformation of the parameter $x$ is required to match the definition domain of input random variables:

$$
z_{i}=\frac{a_{U}-a_{L}}{2} x_{i}+\frac{a_{L}+a_{U}}{2} \quad\left(\text { as } x_{i} \in[-1,1] \text { and } i=1, \ldots, d\right)
$$

and parameters $a_{L}$ and $a_{U}$ are empirically defined as 

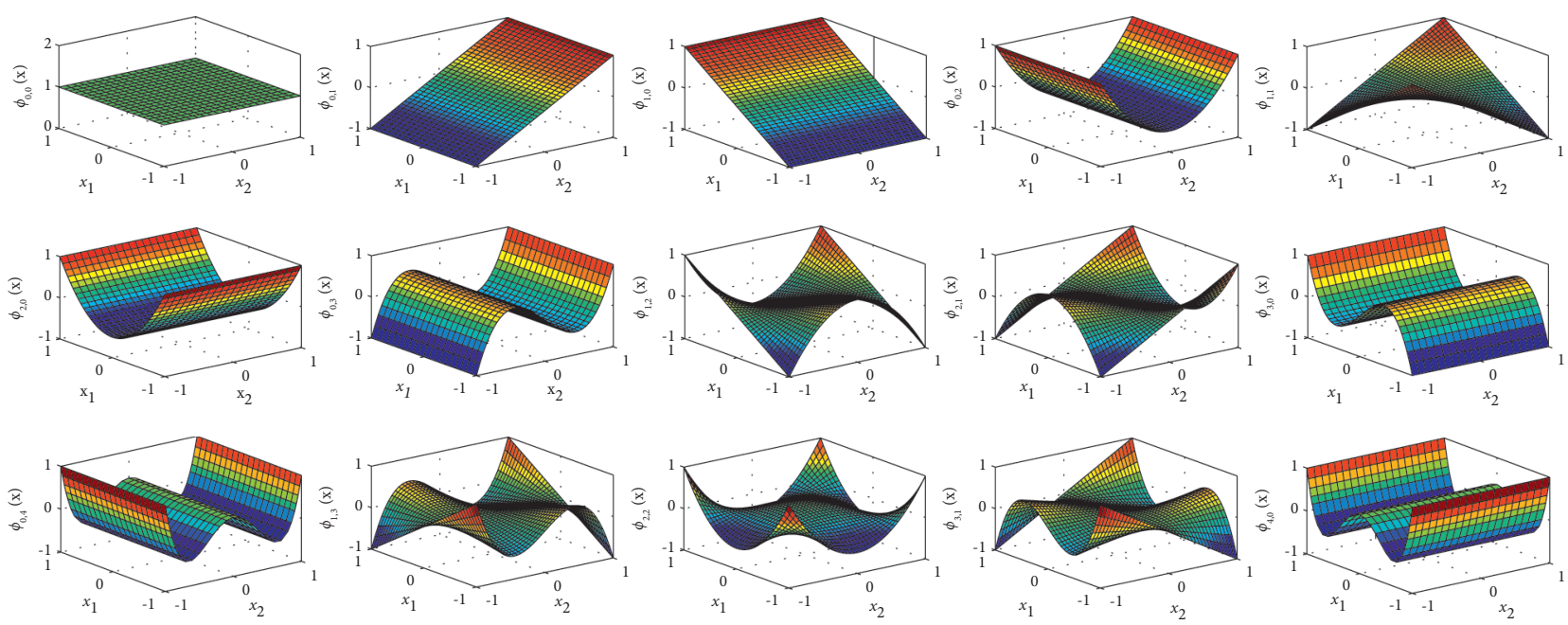

FIgURe 2: Bivariate Gegenbauer polynomials defined by parameters $\beta=1 / 2$ and the polynomial degree $p=4$.

$$
\left\{\begin{array}{l}
a_{L}=F^{-1}\left(\mu_{i}-k \sigma_{i}\right), \\
a_{U}=F^{-1}\left(\mu_{i}-k \sigma_{i}\right),
\end{array}\right.
$$

wherein $F^{-1}(\cdot)$ represents the inverse cumulative distribution function (iCDF) of the random variable $X_{i}$ associated with the mean $\mu_{i}$ and the standard deviation $\sigma_{i}$.

The parameter $k$ is expected to connect with the truncated probability in equation (14). If $X_{i}$ is a Gaussian variable, the truncated probability $\operatorname{Pr}\left[X_{i} \leq a_{L}\right]+\operatorname{Pr}\left[X_{i} \geq a_{U}\right]$ will be less than $0.3 \%$ and $0.01 \%$, respectively, as $k=3$ and 4 . Therefore, the linear transformation in equation (13) with the parameter $k=4$ will be utilized in numerical examples to tackle nonuniformly distributed and skewed random variables.

\section{The Gegenbauer Polynomial-Based Sparse Surrogate Model}

Structural reliability simulations are always realized based on a multivariate performance function and its surrogate model. Once the surrogate model is numerically available, the subsequent reliability analysis can be alternatively realized by the brutal Monte Carlo simulation. Note that the total number of functional evaluations is limited to the development of the surrogate model $\hat{g}(\cdot)$, rather than all random samples. As a comparison, the original random simulation based on $g(\mathbf{X})$ will require $N_{M C S} \geq 100 / P_{F}$ samples [33]. The large number of model repetitions implies a huge amount of computational cost. Therefore, the paper proposes utilizing the chaotic Gegenbauer polynomials to build the surrogate model at first, and the total number of mechanistic model reevaluations will be only limited to the number of numerical operations to develop the surrogate model, rather than directly carrying out the physical modelbased random simulations.

Conventional response surface models usually contain hundreds or even thousands of polynomial terms for a robust estimation result. The global sensitivity analysis result has shown that a relatively small number of component functions is capable of accurately approximating a complex performance function $g(\cdot)$; that is, a sparse approximation is available for the structural reliability analysis. Based on the consideration, the section presents utilizing the chaotic Gegenbauer polynomial-based sparse model to mimic the true performance function. The principle polynomials among all potential explanatory elements are detected based on a small number of model simulation results. To begin with, a standard procedure for the surrogate model developed based on the statistical regression method is briefly summarized as follows.

3.1. The Regression-Based Surrogate Model. To begin with, the set $\zeta=\left\{\mathbf{x}^{(1)}, \ldots, \mathbf{x}^{(n)}\right\}$ consists of $n$ realizations of input random vector $\mathbf{X}$ generated based on a low-discrepancy sequence, for example, the Sobol', the Helton, or the Hammersley algorithm, whereas the corresponding realizations of the chaotic Gegenbauer polynomial set $\left\{\phi_{i}(\mathbf{x})\right\}_{i=0}^{N-1}$ are expressed in a matrix form as

$$
\xi=\left[\begin{array}{cccc}
\phi_{0}\left(\mathbf{x}^{(1)}\right) & \phi_{1}\left(\mathbf{x}^{(1)}\right) & \cdots & \phi_{N-1}\left(\mathbf{x}^{(1)}\right) \\
\phi_{0}\left(\mathbf{x}^{(2)}\right) & \phi_{1}\left(\mathbf{x}^{(2)}\right) & \cdots & \phi_{N-1}\left(\mathbf{x}^{(2)}\right) \\
\vdots & \vdots & \ddots & \vdots \\
\phi_{0}\left(\mathbf{x}^{(n)}\right) & \phi_{1}\left(\mathbf{x}^{(n)}\right) & \cdots & \phi_{N-1}\left(\mathbf{x}^{(n)}\right)
\end{array}\right]_{n \times N} .
$$

Following the theory of multivariate "linear" regression [34], a response surface model for the performance function $g(\cdot)$ can be obtained as

$$
\widehat{y}=\sum_{i=0}^{N-1} b_{i} \phi_{i}(\mathbf{x})+\varepsilon .
$$

Note that the vector $\mathbf{b}=\left[b_{0}, b_{1}, \ldots, b_{N-1}\right]^{T}$ consists of all regression coefficients that are attached to explanatory variables $\left\{\phi_{i}(\mathbf{x})\right\}_{i=0}^{N-1}$. In addition, the residual error is expressed as

$$
\mathbf{r}=\mathbf{y}-\xi \widehat{\mathbf{b}} \text {. }
$$


Here, the model response vector $\mathbf{y}=\left[g\left(\mathbf{x}^{(1)}\right), \ldots, g\left(\mathbf{x}^{(n)}\right)\right]^{T}$ comprises total $n$ realizations of the mechanistic model by continuously feeding each element of the training dataset $\zeta$. And the unknown regression coefficients $\widehat{\mathbf{b}}$ can be expressed in terms of the training dataset $\xi$ as

$$
\widehat{\mathbf{b}}=\left(\boldsymbol{\xi}^{T} \boldsymbol{\xi}\right)^{-1} \boldsymbol{\xi}^{T} \mathbf{y} .
$$

Therefore, once the training matrix $\xi$ defined in equation (15) and corresponding structural response samples $\mathbf{y}$ are numerically available, the least-square minimization based on the 2-norm of the residual error vector $\mathbf{r}$ allows deriving an empirical surrogate model noted in equation (16) for reliability analysis of the structural system.

3.2. A Sparse Regression Model Based on the OMP Algorithm. A fundamental problem in structural reliability analysis signal processing is to develop a reliable sparse surrogate model for multivariate input uncertainties. The orthogonal matching pursuit (OMP) algorithm for the signal processing is presented with the basis dictionary defined by the chaotic Gegenbauer polynomials.

Given a realization of the polynomial parameter $p$, the full dictionary set can be initialized as $\phi_{(0)}=\left[\phi_{0}(\mathbf{x}), \phi_{1}\right.$ $\left.(\mathbf{x}), \ldots, \phi_{N-1}(\mathbf{x})\right]^{T}$, together with principle components $\psi_{(0)}=\varnothing$. Based totally on $n$ training samples in $\zeta$ generated based on the Halton or the Sobol' low-discrepancy scheme, the corresponding model responses $y$ and the polynomial matrix $\xi$ in equation (15) are prepared for subsequent model evaluations. Specifically, the residual error is initialized as $\mathbf{r}_{(0)}=\mathbf{y}$, whereas the significant elements in $\xi$ will be $\chi_{(0)}=\varnothing$.

For a realization of the iterative counter $k \geq 1$, one could implement the OMP-based sparse regression analysis as follows:

(1) Detect the most principle polynomial $\phi_{i^{*}}(\mathbf{x}) \in \psi_{(k-1)}$ based on the following criterion:

$i^{*}=\arg \max \left|\xi_{i}^{T} \mathbf{r}_{(k-1)}\right| \quad($ as $i=0, \ldots, N-1)$,

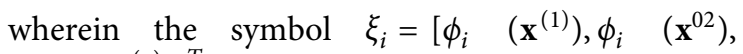
$\left.\ldots, \phi_{i}\left(\mathbf{x}^{(n)}\right)\right]^{T}$ denotes an $i$ th column of the polynomial matrix $\xi$ that is evaluated based on $n$ samples of the input random vector $\mathbf{X}$. Therefore, the polynomial index parameter for an $i^{*}$ th significant polynomial will be updated as $\psi_{(k)}=\psi(k-1) \cup$ $\left\{\phi_{i^{*}}(\mathbf{x})\right\}$ and $\chi_{(k)}=\chi_{(k-1)} \cup\left\{\xi_{i^{*}}\right\}$, respectively. Meanwhile, the explanatory dictionary is revised by excluding the element $\phi_{i^{*}}(\mathbf{x})$, that is, $\left.\phi_{(k)}=\phi_{(k}-1\right) \ominus$ $\left\{\phi_{i^{*}}(\mathbf{x})\right\}$ for subsequent iterations.

(2) Update the model result by calculating the coefficients $\widehat{\mathbf{b}}_{(k)}=\left[\chi_{(k)}^{T} \chi_{(k)}\right]^{-1} \chi_{(k)}^{T} \mathbf{y}$, which determines the model after a $k$ th iteration as

$$
\widehat{y}_{(k)}=\psi_{(k)}^{T} \widehat{\mathbf{b}}_{(k)} .
$$

Note that the residual error will be $\mathbf{r}_{(k)}=(\mathbf{I}-\mathbf{A}) \mathbf{y}$, and $\mathbf{A}=\chi_{(k)}\left[\chi_{(k)}^{T} \chi_{(k)}\right]^{-1} \chi_{(k)}^{T}$, and the symbol I denotes an $n \times n$ identity matrix.

(3) Evaluate the sparse regression model in equation (20) based on stopping criteria listed in Table 1. Or else, set $k=k+1$ and repeat Steps $1-3$ till the largest realization of the iterative counter $N_{\max }=\operatorname{rank}(\xi)$.

It is observed that the OMP algorithm is a stepwise forward greedy algorithm to select principle components. In this regard, a main issue for the sparse regression can stop the greedy selection process at "a right time." However, stopping rules of the greedy algorithm are mainly defined by mathematical characteristics of the residual error. Typical realizations include the $\ell_{2}$ and $\ell_{\infty}$ measures, that is, $\left\|\mathbf{r}_{(k)}\right\|_{2} \leq \varepsilon_{1}$ and $\left\|\psi_{(k)}^{T} \mathbf{r}_{(k)}\right\|_{\infty} \leq \varepsilon_{2}$, wherein, parameters $\varepsilon_{i}$ (as $i=1,2$ ) denote the predefined error thresholds. In addition, a relative-error-based measure can also be used:

$$
\frac{\left\|\mathbf{r}_{(k-1)}-\mathbf{r}_{(k)}\right\|_{\infty}}{\left\|\mathbf{r}_{(k)}\right\|_{\infty}}<\varepsilon_{3},
$$

which is evaluated by two successive residual errors $\mathbf{r}_{(k-1)}$ and $\mathbf{r}_{(k)}$ (as $\left.k=1,2, \ldots\right)$ in model iterations. Note that the $\ell_{\infty}$-norm used here is much more strict than criteria based on the $\ell_{2}$ norm.

Rather than the hard stopping criteria that are evaluated directly based on the residual error term $\mathbf{r}_{(k)}$, an $F$-valuebased soft stopping criterion can be further used to exclude incorrectly identified component functions. This is because the multivariate Gegenbauer polynomials might not be exactly orthogonally defined with each other due to the truncated simulation domain $\Omega=\cup_{i} \Omega_{i}$ as shown in equation (14). It is highly possible that the residual error $\mathbf{r}_{(k)}$ cannot be exactly represented by the remaining polynomial set after a $k$ th iteration. This is the motivation to utilize an $F$-test-based procedure to exclude spurious basis functions and maintain the high sparsity of the response surrogate model.

Recall that the covariance matrix for regression coefficients $\widehat{\beta}_{(k)}$ is estimated as

$$
\operatorname{Cov}\left[\widehat{\boldsymbol{\beta}}_{(k)}\right]=\sigma^{2}\left[\boldsymbol{\chi}_{(k)}^{T} \boldsymbol{\chi}_{(k)}\right]^{-1}
$$

wherein $\sigma^{2}$ denotes the variance of the error term $\epsilon$, and its unbiased estimator is

$$
\widehat{\sigma}^{2}=\frac{\mathbf{r}_{(k)}^{T} \mathbf{y}}{n-M}
$$

Herein, the integers $n$ and $m$ represent the total number of training samples and polynomial elements in $\widehat{\beta}_{(k)}$, respectively. Then, an $F$-statistics for the significant test of regression coefficients are defined as

$$
F_{j}=\frac{\widehat{b}_{j}^{2}}{\left[\operatorname{Cov}\left(\widehat{\mathbf{b}}_{(k)}\right)\right]_{j j}} \quad\left(\text { for each } \widehat{b}_{j} \in \widehat{\mathbf{b}}_{(k)} \text { and } j=1, \ldots, M\right) \text {, }
$$


TABLE 1: Stopping criteria used in numerical simulations.

\begin{tabular}{llll}
\hline & Hard rules & Soft rule \\
\hline$\left\|\mathbf{r}_{(k)}\right\|_{2} \leq \varepsilon_{1}$ & $\left\|\psi_{(k)}^{T} \mathbf{r}_{(k)}\right\|_{\infty} \leq \varepsilon_{2}$ & $\left\|\mathbf{r}_{(k-1)}-\mathbf{r}_{(k)}\right\|_{\infty} /\left\|\mathbf{r}_{(k)}\right\|_{\infty} \leq \varepsilon_{3}$ & $F_{j^{*}}<F_{\alpha^{*}}$ \\
\hline
\end{tabular}

which will be used to detect the most insignificant coefficient $\widehat{b}_{j^{*}}$ with the minimum $F$-value:

$$
j^{*}=\operatorname{argmin}\left\{F_{j}, j=1, \ldots, M\right\} .
$$

Together with the significant level $\beta^{*}$ in numerical examples, the $F$-value-based "soft" stopping criterion will be numerically implemented depending on the following cases:

Case 1: $F_{j^{*}}<F_{\beta^{*}}(1, n-M)$ and $i^{*}=j^{*}$. This implies that the null hypothesis $\left\{H_{0}: \widehat{\beta}_{i^{*}}=0\right\}$ cannot be rejected based on the $F$ test for the $\widehat{\beta}_{i^{*}}$ th regression coefficient, and the surrogate model $\widehat{y}_{(k-1)}=\phi_{(k-1)} \widehat{\beta}_{(k-1)}$ is the sparse regression result for the performance function $g(\cdot)$.

Case 2: $F_{j^{*}}<F_{\beta^{*}}(1, n-M)$ but $i^{*} \neq j^{*}$. One needs to update the significant polynomial set $\phi_{(k)}$ by excluding the spurious principle component $\phi_{j^{*}}(\mathbf{x})$ and recalculate regression coefficients $\widehat{\beta}_{(k)}$ for afterward significant tests.

Otherwise, go to Step 3 till the counting variable $k$ reaches its upper bound $N_{\max }=\operatorname{rank}(\xi)$.

In summary, all considered hard and soft stopping criteria are listed in Table 1 . In numerical simulations, a total of eight cases about realizations of parameters $\varepsilon_{i}$ and $\alpha^{*}$ will be considered, that is, $\varepsilon_{1}=0.1,0.05$ (Cases 1 and 2), $\varepsilon_{2}=$ $0.1,0.05$ (Cases 3 and 4), $\varepsilon_{3}=0.1,0.05$ (Cases 5 and 6), and $\alpha^{*}=0.05,0.01$ (Cases 7 and 8) given a realization of $\varepsilon_{i}$ and $\alpha^{*}$. Together with several examples in the literature, potential applications of the Gegenbauer polynomial-based surrogate model for structural reliability analysis will be demonstrated below.

\section{Numerical Assessments of the Gegenbauer Polynomial-Based Surrogate Model}

This section examines the performance of the chaotic Gegenbauer polynomial-based sparse regression method for structural reliability analysis based on several examples in the literature. In this regard, an accuracy measure of the surrogate model is defined as

$$
\text { regression error }=\frac{\sum_{i=1}^{N_{\text {Test }}}\left[g\left(\mathbf{x}^{(i)}\right)-\hat{g}\left(\mathbf{x}^{(i)}\right)\right]^{2}}{\sum_{i=1}^{N_{\text {Test }}}\left[g\left(\mathbf{x}^{(i)}\right)-\bar{y}\right]^{2}}
$$

wherein the mean value of the model response is estimated as $\bar{y}=1 / N_{\text {Test }} \sum_{i=1}^{N_{\text {Test }}} g\left(\mathbf{x}^{(i)}\right)$ based on $N_{\text {Test }}$ realizations of the input random vector $\mathbf{X}$. In addition, a model sparsity indicator is defined as

$$
\text { ratio of sparsity }=\frac{\text { the number of detected significant terms }(M)}{\text { total number of elements in }\left\{\phi_{0}(\mathbf{x}), \ldots, \phi_{N-1}(\mathbf{x})\right\}} \times 100 \% \text {, }
$$

and a small value of the ratio of the sparsity (RoS) (e.g., $\leq 20 \%$ ) is expected in engineering realities to reduce the total number of training samples for structural reliability analysis.

4.1. The Ishigami function. The mathematical example investigates the numerical performance of the chaotic Gegenbauer polynomial in sparse regressions analysis by considering the Ishigami function:

$$
g(\mathbf{X})=\sin \left(X_{1}\right)+a\left[\sin \left(X_{2}\right)\right]^{2}+b \sin \left(X_{1}\right) X_{3}^{4} .
$$

All input variables $X_{i}$ are i.i.d. uniform random variables within the interval $[-\pi, \pi]$, and constants $a$ and $b$ are assumed as 7.0 and 0.1 , respectively, in the literature. Note that the full model consists of 286 terms for the highest polynomial order $p=10$.

Since the analytical result of the Ishigami function is available, a numerical experiment is carried out to examine the effect of various stopping criteria for uncertainty analysis of the Ishigami function. With 200 random realizations of the input vector $\mathbf{X}$, the proposed algorithm is followed to determine dominant basis functions and the corresponding sparse regression model.
Figure 3 presents simulation results of the regression error $(\mathrm{Re})$ and the ratio of sparsity $(\mathrm{RoS})$ for various realizations of the polynomial parameter $\beta \in[-0.8,0.8]$ for $X_{1}$ and $X_{2}$, whereas investigated stopping criteria in Table 1 will be investigated. This allows one to determine a better stopping criterion for small realizations of the structural performance function. Results have justified that the polynomial parameter $\beta$ can determine divergent surrogate models in terms of the approximation error and the sparsity ratio. Specifically, realizations $\beta=-0.6$ and -0.69 determine the minimal error and the sparsity ratio results for the uniform random variables, respectively. Besides, an increase in the absolute value of $|\beta|$ will generally increase the global errors and the sparsity ratio. In this regard, the Chebyshev polynomial determined by the parameter $\beta=0$ will be further examined for various structural reliability problems.

Specifically, the effect of the parameter $\beta$ on the numerical accuracy of the surrogate model is further investigated by considering $\beta$ varying from -0.2 to 0.2 with an incremental Step 0.05. Results in Figure 4 show that there are no significant differences among prediction error results, which are less than $3 \times 10^{-6}$ for all realizations cases, yet the cases $\beta=-0.05$ and $\beta=0$ can determine relatively smaller sparsity, that is, $\leq 6.7 \%$. 


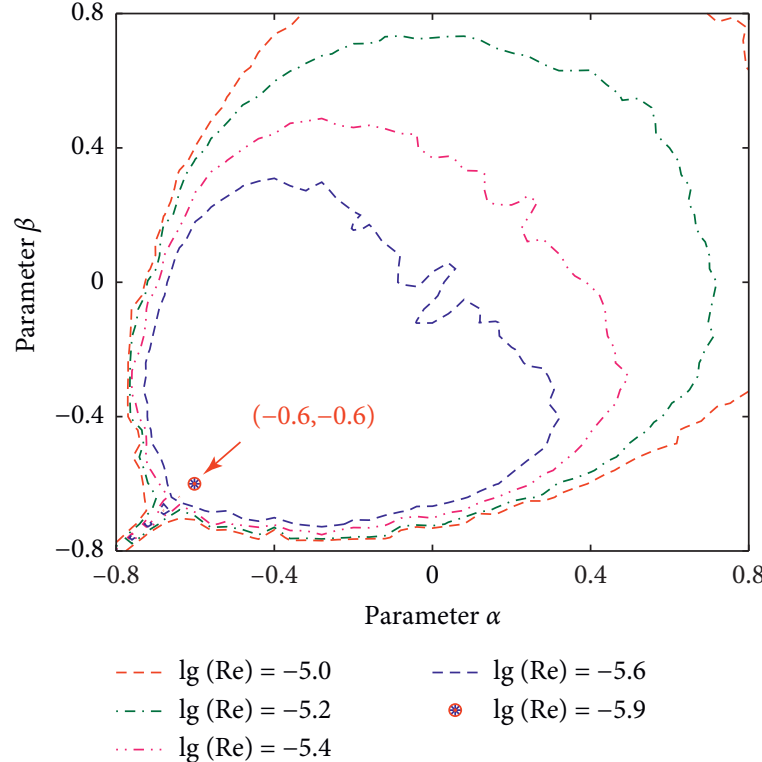

(a)

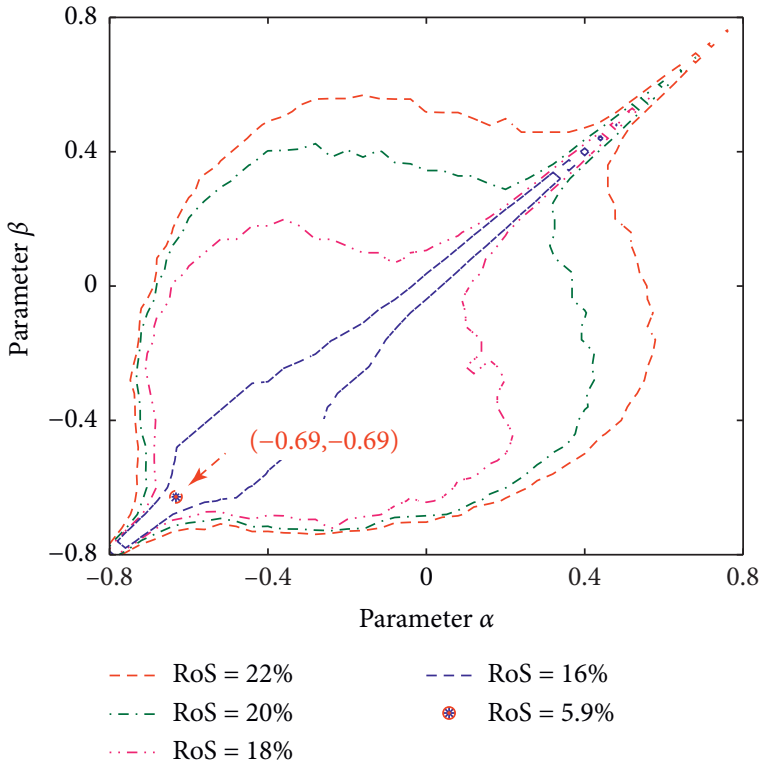

(b)

FIGURE 3: The isoline for the median values of the regression error $(\operatorname{Re})$ and the sparsity ratio as $\left.\beta_{1}, \beta_{2} \in[-0.8,0.8]\right)$. (a) The regression error. (b) The sparsity ratio.

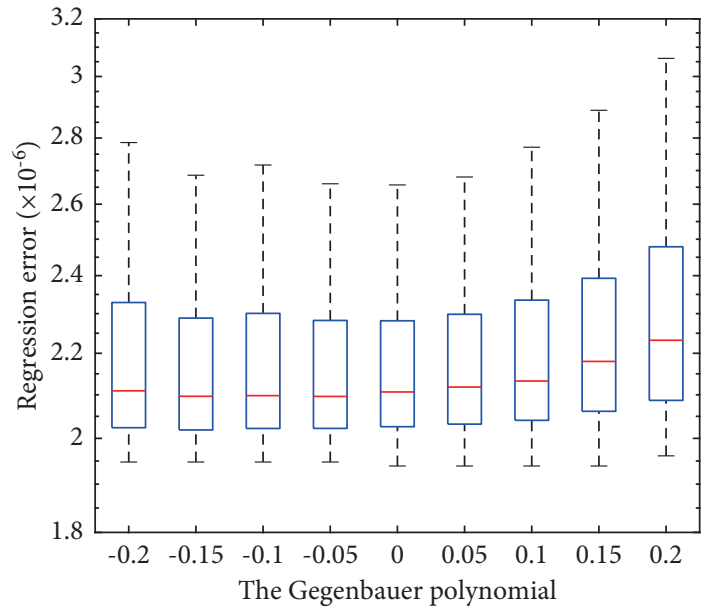

(a)

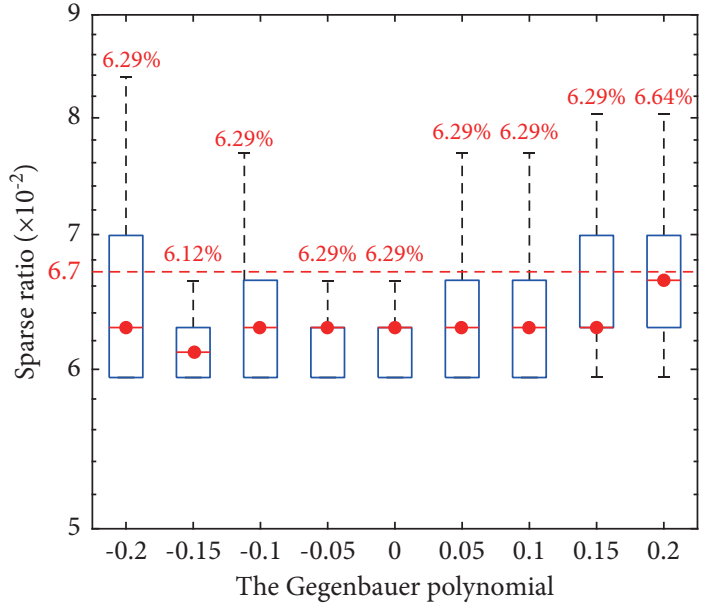

(b)

Figure 4: Results for the regression errors and the sparse ratio as the polynomial parameter $\beta \in[-0.2,0.2]$. (a) The regression error. (b) The sparsity ratio.

Therefore, the case $\beta=0$ will be used to check various stopping criteria in Table 1 as follows.

The polynomial parameter is further fixed as $\beta=0$, numerical results of the regression error and the sparsity ratio are estimated for various stopping criteria in Figure 5. It is observed that the global regression error and sparse ratio are much higher than the average result for the hard stop criterion $\left\|\psi_{(k)}^{T} \mathbf{r}_{(k)}\right\| \|_{\infty}$ with the threshold parameter $\varepsilon_{2}=0.05$ (Case 4) and the $F$-value-based soft rule with the parameter $\alpha^{*}=0.05$ (Case 7). Specifically, the largest regression error and the sparse ratio results are observed for
Case 2, that is, $\left\|\mathbf{r}_{(k)}\right\|_{2} \leq 0.05$. In general, the optimum result is determined by the hard rule $\left\|\psi_{(k)}^{T} \mathbf{r}_{(k)}\right\| \|_{\infty} \leq 0.1$ (Case 4) and the soft criterion $F_{\alpha^{*}}=0.05$ (Case 7) for the Ishigami example. The criteria will be further examined by a highdimensional uncertain model as follows.

4.2. The High-Dimensional Function. To further examine the performance of the proposed Gegenbauer polynomial-based regression method for uncertainty analysis of high-dimensional models, the example considers the multivariate function: 


$$
g(\mathbf{X})=3-\frac{5}{d} \sum_{k=1}^{d} k X_{k}+\frac{1}{d} \sum_{k=1}^{d} k X_{k}^{3}+\ln \left[\frac{1}{3 d} \sum_{k=1}^{d} k\left(X_{k}^{2}+X_{k}^{4}\right)\right]
$$

Herein, $X_{i}$ are uniform i.i.d. variables within the region $[1,2]$, and various realizations of the dimensionality parameter $d$ are considered.

To examine the performance of the proposed sparse regression approach for a problem with various dimensions of the input random vector $\mathbf{X}$, the dimensionality parameter $d$ is generally assumed as 10 and 20 . Combining the polynomial parameter $\beta=0$ and various stopping criteria, Figures 6 and 7 summarize simulation results for the global regression error and the sparsity ratio associated with the dimensionality parameters $d=10$ and 20 .

Results have shown that the stop criterion $\left\|\psi_{(k)}^{T} \mathbf{r}_{(k)}\right\| \|_{\infty} \leq 0.1$ (Case 4) and the $F$ statistic-based criteria in Cases 7 and 8 are able to determine relatively small results of the regression error and the sparsity ratio. Specifically, the criterion (Case 2), that is, $\left\|\mathbf{r}_{(k)}\right\|_{2} \leq 0.05$, cannot determine the surrogate model as accurate as other rules if $d=20$. Consider a better balance of the global error and the sparsity ratio achieved by the hard criteria $\left\|\psi_{(k)}^{T} \mathbf{r}_{(k)}\right\| \|_{\infty} \leq 0.1$ and the soft rule $F_{j^{*}}<F_{\alpha^{*}}$; they will be used to build the surrogate model for structural reliability analysis in the following simulations.

To further examine the utility of various polynomials in the model approximation, the Chebyshev polynomial of the first kind, that is, the polynomial parameter $\beta=-1 / 2$, is further used to build a surrogate model of the high-dimensional function. Results in Figure 8 have shown that the proposed regression method is rather effective for the highdimensional problem, for example, the dimensionality parameter $d \geq 15$. The corresponding sparsity ratio is less than $10 \%$, and the regression errors are in the magnitude of $10^{-5}$. The high accuracy ensures the numerical effectiveness of the surrogate model for structural reliability simulations.

Figure 9 finally presents numerical results for the response probability density function of the high-dimensional function. With 500 Sobol' sequences to build the sparse model at first, the brutal-force MCS is fully realized based on the surrogate model $\hat{g}(\mathbf{X})$. The close agreement of simulation results between the surrogate and the true models has confirmed the high accuracy of the proposed approach.

4.3. Reliability Analysis of a Steel Frame Structure. The example further demonstrates potential applications of the proposed Gegenbauer polynomial-based sparse regression method for reliability analysis of a two-bay six-story steel frame. As depicted in Figure 10, random variables of the frame structure include the modulus of elasticity $E$, the moment of inertia $I$, and structural external loads $P_{i}(i=1, \ldots, 6)$. The probabilistic characteristics of input random variables are summarized in Table 2 , whereas the performance function is defined as the structural maximal interstorey drift over the limit $u_{0}=11 \mathrm{~cm}$ :

$$
\begin{aligned}
g(\mathbf{X}) & =u_{0}-D_{\max }(\mathbf{X}) \\
& =u_{0}-\max \left\{\left|d_{A}(\mathbf{X})-d_{D}(\mathbf{X})\right|,\left|d_{B}(\mathbf{X})-d_{E}(\mathbf{X})\right|,\left|d_{C}(\mathbf{X})-d_{F}(\mathbf{X})\right|\right\}
\end{aligned}
$$

Herein, $D_{\max }$ denotes the maximal interstorey drift, whereas $d_{(\text {.) }}$ represents the nodal lateral displacements that are explicitly evaluated based on a finite element (FE) model of the steel frame structure.

To determine the failure probability of the steel frame structure, the Gegenbauer polynomial with the parameter $\beta=0$ is used to develop a sparse surrogate model with the polynomial order $p=3$ and the sample size $n=500$. Note that a similar result based on the parameter $\beta=1 / 2$ is determined, yet omitted here for the sake of brevity.

Figure 11(a) depicts numerical results for the empirical PDF of the structural maximum roof drift, and 500 samples are sufficient to determine a surrogate model to mimic the true performance function. The POE result pictured in Figure 11(b) provides the estimation of the structural failure probability as $1.44 \times 10^{-2}$, which is fairly close to the benchmark result $1.49 \times 10^{-2}$ in [35].

To further evaluate the robustness of the proposed approach, 100 repetitions of the sparse regression analysis have been implemented, and the corresponding results for the structural failure probability are summarized in Figure 12. Since each round of the sparse regression analysis only requires 500 nonintrusive model runs. It has exhibited the high efficiency of the proposed approach for the structural reliability analysis, whereas the small standard deviation result $\left(3.74 \times 10^{-4}\right)$ confirms the robustness of the proposed method as well.

4.4. Reliability Analysis of a Bar Structure with Spatially Varying Stochastic Material Properties. This section illustrates an application of the proposed approach by considering a bar structure with spatially varying Young's modulus. As depicted in Figure 13, a bar structure with unit length and cross-sectional area (i.e., $L=1$ and $A=1$ ) is subjecting axially distributed load $P(x)=x$. Specifically, the modulus of elasticity $E$ is model via a homogeneous Gaussian random field:

$$
\operatorname{Cov}\left(x, x^{\prime}\right)=\sigma_{E}^{2} \exp \left(-\frac{\left|x-x^{\prime}\right|}{\delta}\right) \text { with } x, x^{\prime} \in[0, L],
$$

which is the exponential covariance function and symbols $x$ and $x^{\prime}$ represent any two positions along the bar. The parameters $\sigma_{E}=0.1$ unit and $\delta$ denote the standard deviation and correlation length of the Gaussian random field, respectively. 


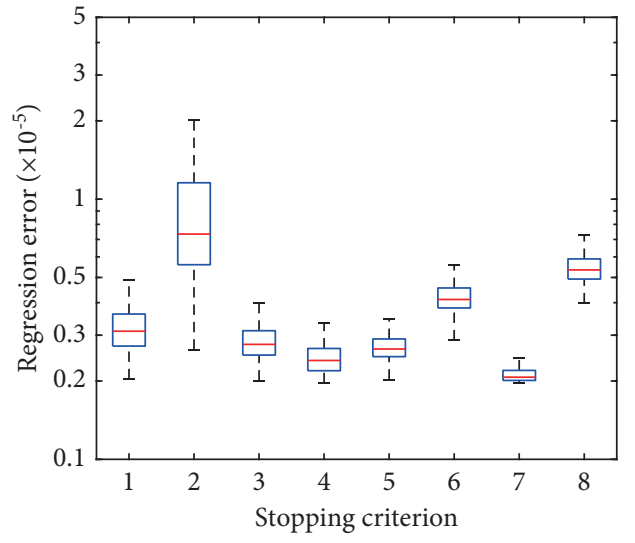

(a)

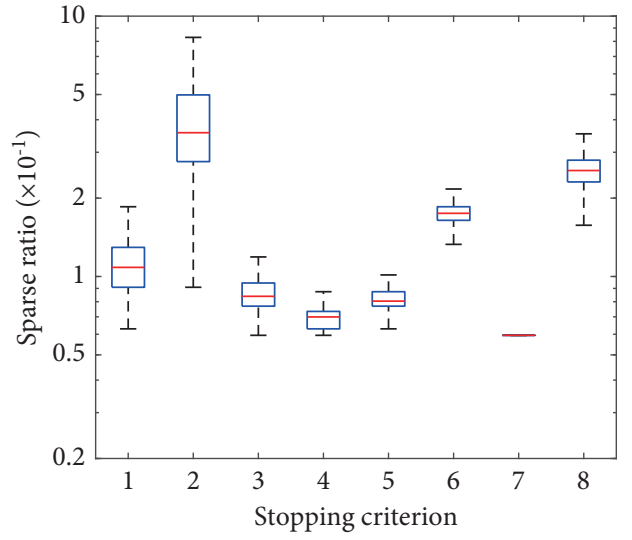

(b)

FIgURE 5: Results for the regression errors and the sparse ratio. (a) The regression error. (b) The ratio of the sparsity.

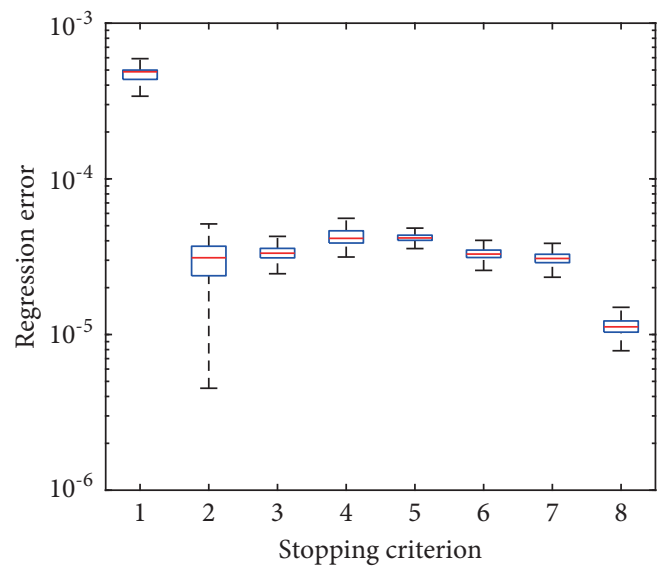

(a)

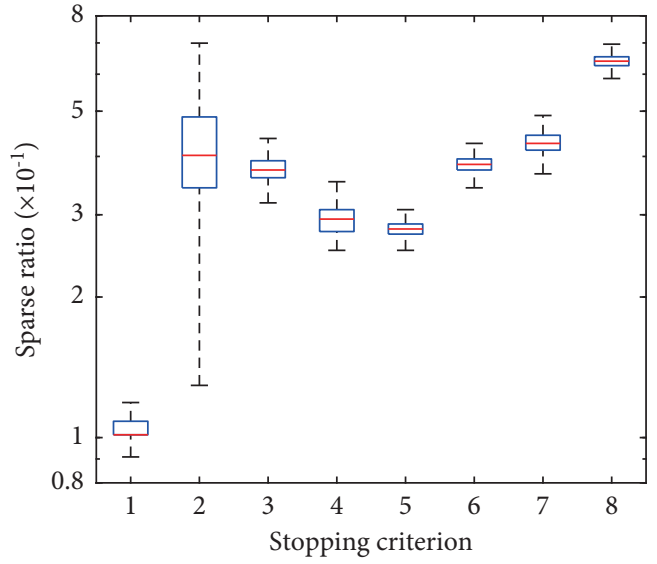

(b)

FIgURE 6: Results for the regression error and the sparsity ratio for the case $d=10$. (a) The regression error. (b) The sparsity ratio.

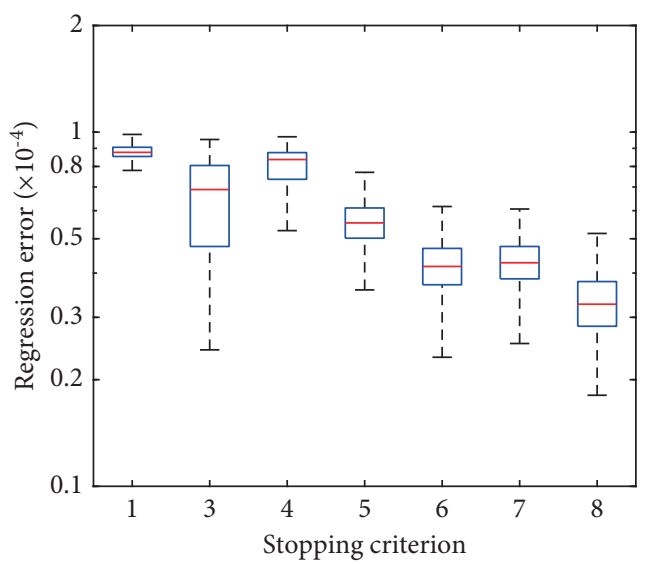

(a)

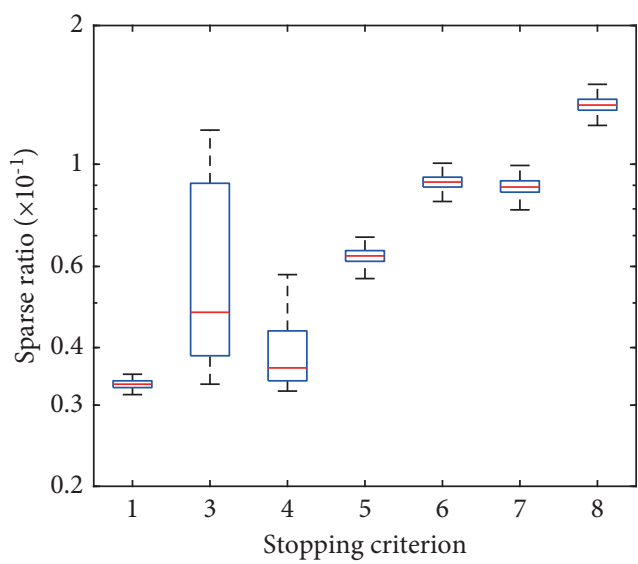

(b)

FIgURE 7: Results for the regression error and the sparsity ratio for the case $d=20$. (a) The regression error. (b) The sparsity ratio. 


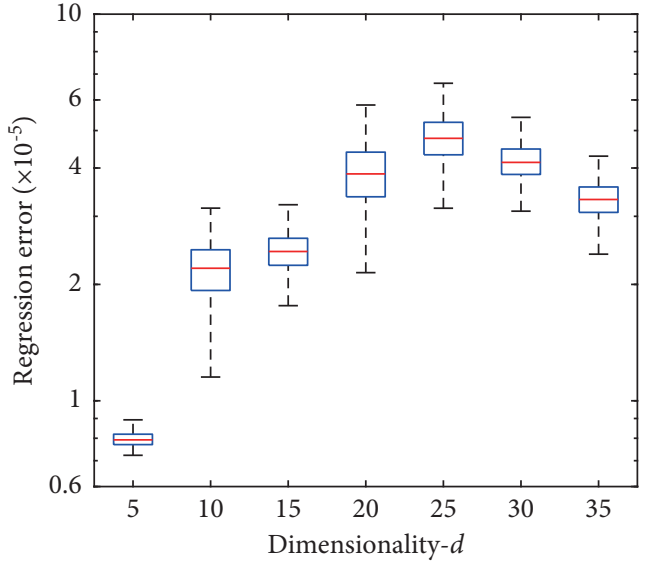

(a)

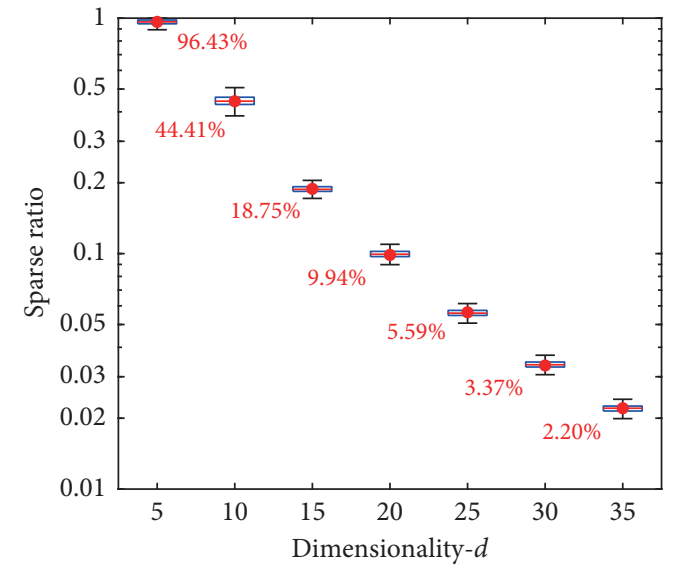

(b)

FIgURE 8: Results for the regression error and the sparsity ratio of the Gegenbauer polynomial-based surrogate model. (a) The regression error. (b) The sparsity ratio.

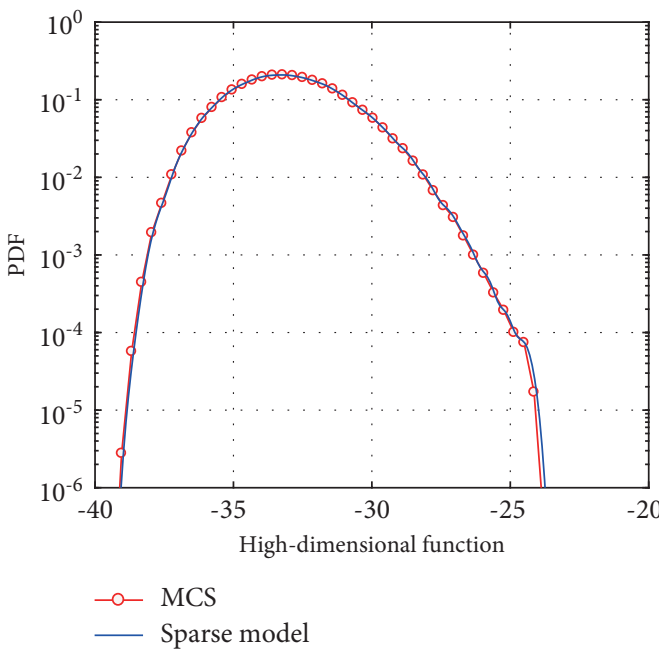

(a)

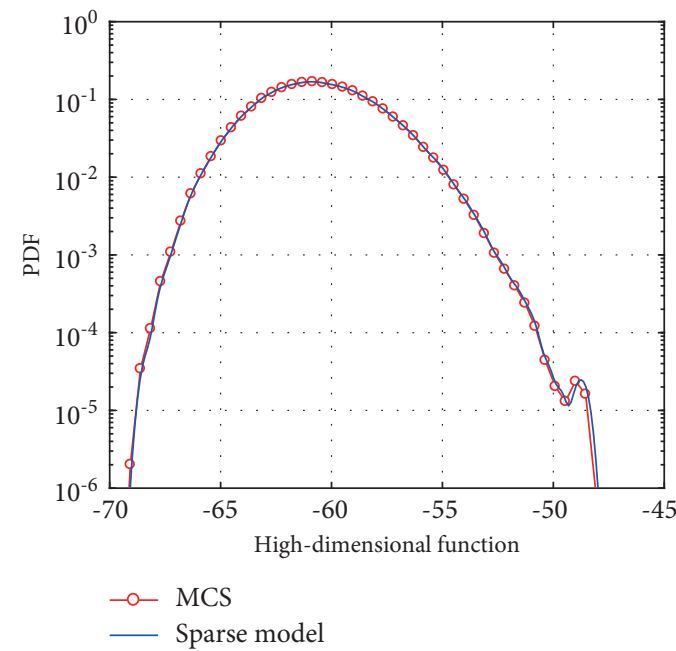

(b)

FIGURE 9: Results for response probability density functions (PDFs) of sparse Gegenbauer polynomial model with parameter $\beta=0$. (a) Semilog plot of PDF: $d=20$. (b) Semilog plot of PDF: $d=35$.

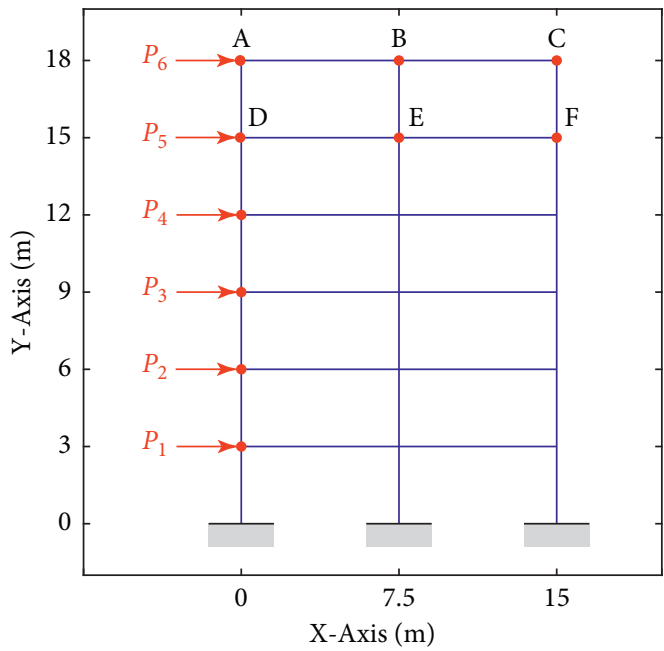

Figure 10: A steel frame structure subjected to lateral loads. 
TABLE 2: Random variables of the steel frame structure.

\begin{tabular}{llllll}
\hline Symbol & Variable & Unit & Mean value & COV & Distribution \\
\hline$X_{1}$ & $E_{b}$ & $\mathrm{~N} / \mathrm{m}^{2}$ & $2 \times 10^{10}$ & 0.10 & Log-normal \\
$X_{2}$ & $I_{b}$ & $\mathrm{~m}^{4}$ & $1 \times 10^{-3}$ & 0.10 & Log-normal \\
$X_{3}$ & $E_{c}$ & $\mathrm{~N} / \mathrm{m}^{2}$ & $2 \times 10^{10}$ & 0.10 & Log-normal \\
$X_{4}$ & $I_{c}$ & $\mathrm{~m}^{4}$ & $1.5 \times 10^{-3}$ & 0.10 & Log-normal \\
$X_{5}$ & $P_{1}$ & $\mathrm{~N}$ & $2.5 \times 10^{4}$ & 0.25 & Normal \\
$X_{6}$ & $P_{2}$ & $\mathrm{~N}$ & $2.8 \times 10^{4}$ & 0.25 & Normal \\
$X_{7}$ & $P_{3}$ & $\mathrm{~N}$ & $2.9 \times 10^{4}$ & 0.25 & Normal \\
$X_{8}$ & $P_{4}$ & $\mathrm{~N}$ & $3.0 \times 10^{4}$ & 0.25 & Normal \\
$X_{9}$ & $P_{5}$ & $\mathrm{~N}$ & $3.1 \times 10^{4}$ & 0.25 & Normal \\
$X_{10}$ & $P_{6}$ & $3.2 \times 10^{4}$ & 0.25 & Normal \\
\hline
\end{tabular}

The subscripts $b$ and $c$ represent beam and column, respectively. COV: the coefficient of variation.

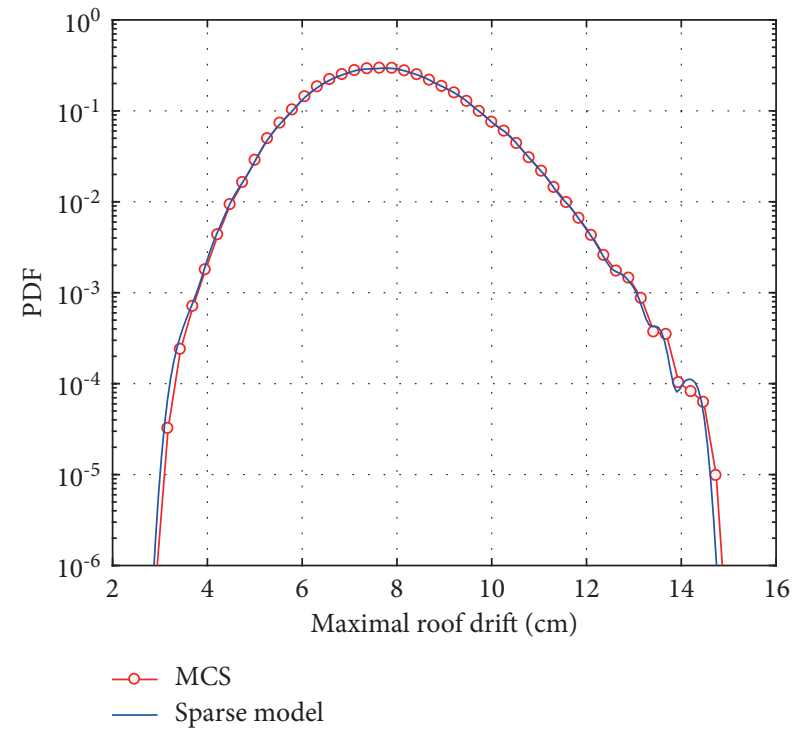

(a)

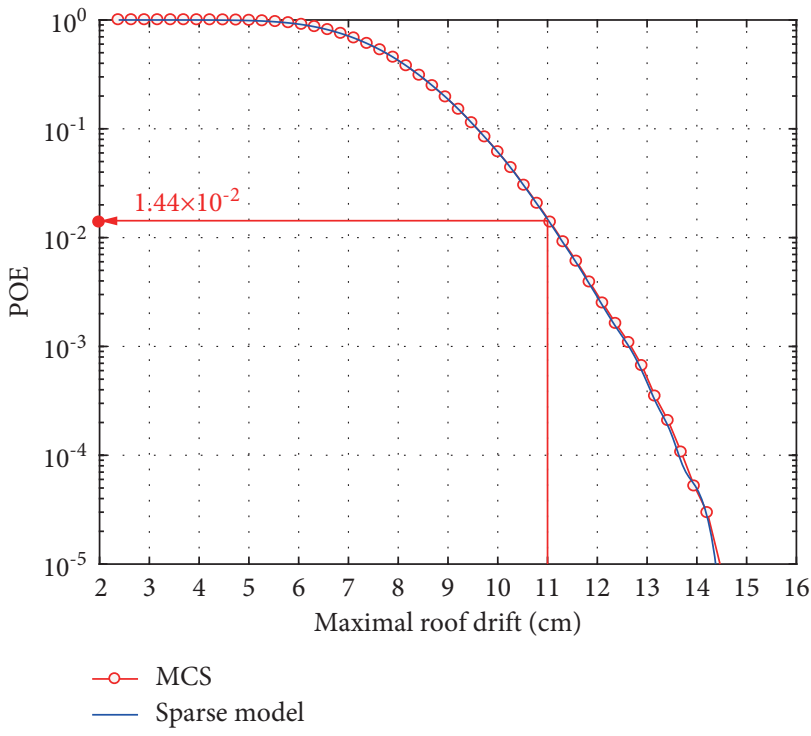

(b)

Figure 11: Results for structural failure probability of the steel frame structure predicted by means of the sparse Gegenbauer polynomial model with parameter $\beta=0$. (a) Probability density function. (b) Probability of exceedance (POE).

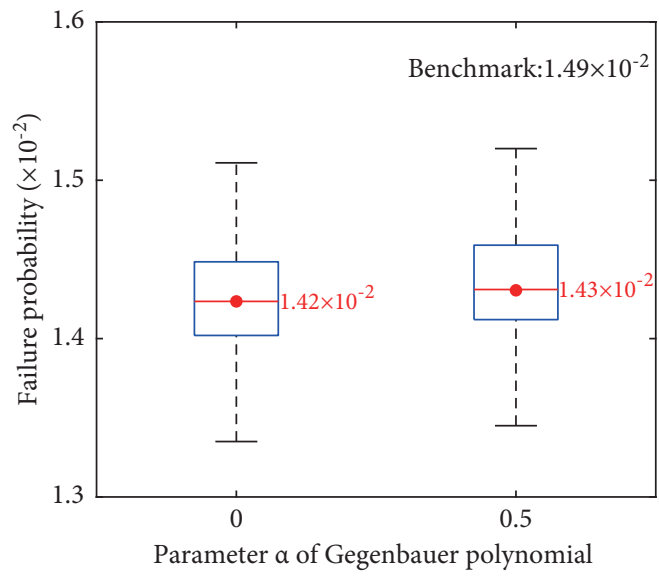

FIGURE 12: Statistical characteristics of the structural failure probability obtained based on 100 repetitions of the sparse regression method. 


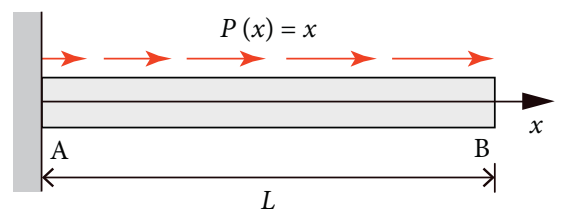

Figure 13: A bar structure under distributed axial load.
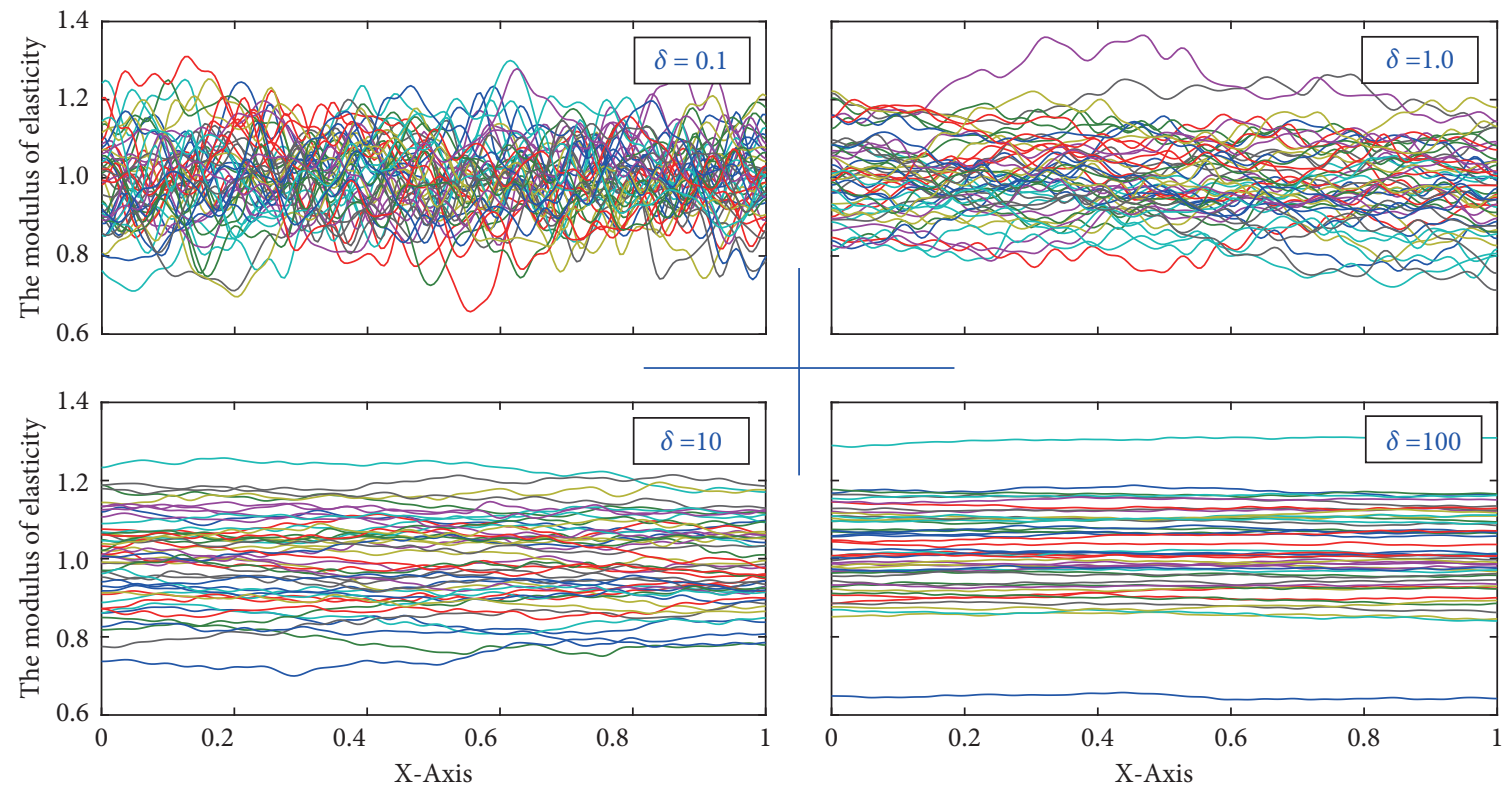

FIGURE 14: Realizations of the modulus elasticity random field with various values of the correlation length parameter $(\delta=0.1,1.0,10$, and $100)$.

Following the theory of the Karhunen-Loève expansion, the material random field is numerically parameterized based on its first- $d$ eigenpairs $\left\{\lambda_{i}, \phi_{i}(x)\right\}(i=1, \ldots, d)[36]$ :

$$
E(x) \approx \mu_{E}(x)+\sum_{i=1}^{d} \sqrt{\lambda_{i}} \phi_{i}(x) X_{i},
$$

wherein $X_{i}$ denote independent standard Gaussian random variables, whereas the mean value of the random field is constantly assumed as $\mu_{E}(x)=1.0$.

Failure events for reliability analysis of the bar structure are defined by the maximum axial displacement $D_{\max }(\mathbf{X})$ over a threshold value of 0.40 :

$$
\text { failure events }=\left\{0.40-D_{\max }(\mathbf{X}) \leq 0\right\} .
$$

Here, random vector $\mathbf{X}=\left[X_{1}, \ldots, X_{d}\right]^{T}$ comprises all Gaussian random variables in equation (32) for numerical discretization of the random field.

With various realizations of the correlation length parameter, that is, $\delta=0.1,1.0,10$, and 100 , numerical realizations of the elasticity random field are presented in Figure 14. Note that ten random variables will be enough to represent more than $95 \%$ of the original variability. This implies that the random vector $\mathbf{X}$ consists of ten standard Gaussian random variables. In addition, a large value of the correlation length parameter (e.g., $\delta=100$ ) increases the statistical dependency between positions of the random modulus elasticity $E(x)$. This allows one to examine the numerical performance of the proposed approach in dealing with dependent input uncertainties.

To implement, the sparse regression method with the polynomial order $p=3$ and 500 Sobol' sequences is employed to develop a surrogate model for the structural maximal axial displacement $D_{\max }(\mathbf{X})$. Results for PDFs of $D_{\max }(\mathbf{X})$ are presented in Figure 15 for various realizations of the correlation length parameter $\delta$. It is observed that the response variability is directly related to the parameter $\delta$ in the random field model, and the case $\delta=100$ determines the largest variation of the structural response quantity.

Based on the structural performance function defined in equation (33), Table 3 summarizes the structural failure probability for various realizations of the correlation length parameter $\delta$. The close agreement between the estimation and benchmark results has verified the high accuracy and numerical efficiency of the sparse regression method.

Figure 16 further summarizes simulation results for the sparsity ratio and the global regression error of the surrogate model. The sparsity ratio result is dramatically decreased with an increase of the correlation parameter $\delta$. A larger value of the parameter $\delta$ implies numerical realizations of the Young's Modulus at two different locations are strongly correlated. The statistical dependency will increase the 


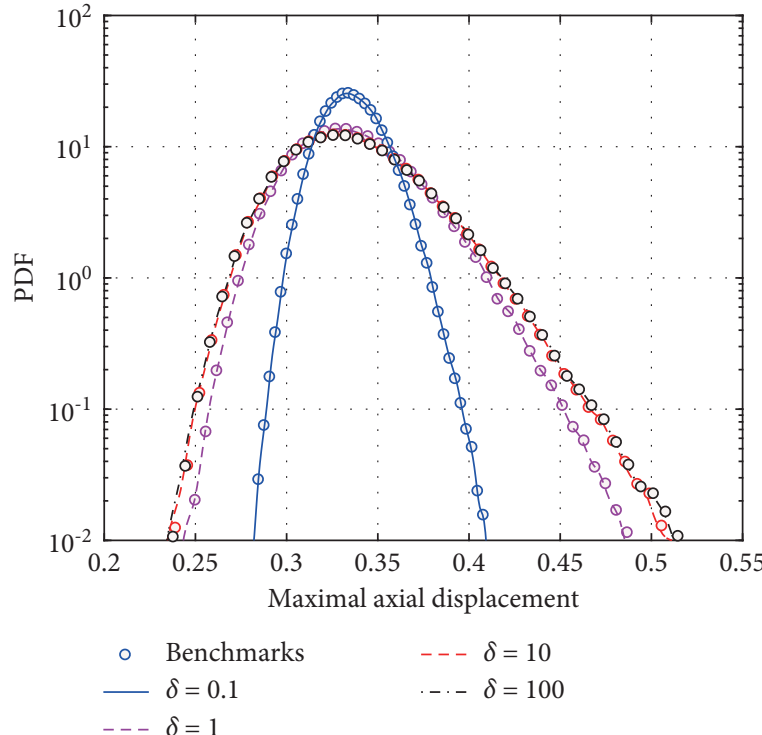

(a)

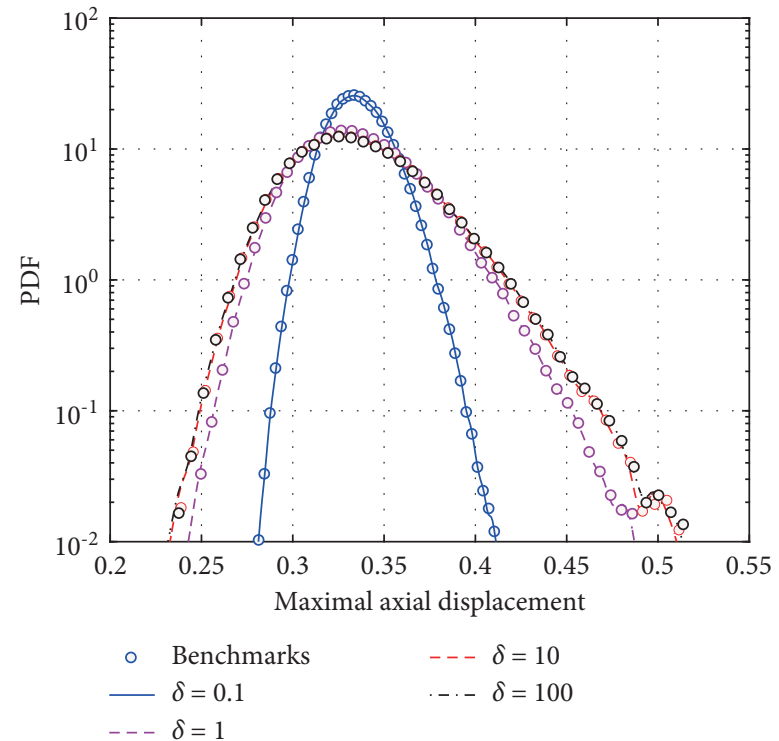

(b)

FIGURE 15: Results for the response distribution of the maximum axial displacement of the bar structure with various correlation length parameters. (a) The sparse surrogate model with the parameter $\beta=0$. (b) The sparse surrogate model with the parameter $\beta=1 / 2$.

TABLE 3: Structural failure probability for various values of the correlation length parameter $(b)$.

\begin{tabular}{lcccc}
\hline & & \multicolumn{2}{c}{ The correlation length parameter } \\
& $b=0.1$ & $b=1.0$ & $b=10$ & $b=100$ \\
\hline MCS & $3.20 \times 10^{-4}$ & $3.09 \times 10^{-2}$ & $4.63 \times 10^{-2}$ & $4.81 \times 10^{-2}$ \\
Sparse model: $\beta=0$ & $2.60 \times 10^{-4}$ & $3.10 \times 10^{-2}$ & $4.66 \times 10^{-2}$ & $4.85 \times 10^{-2}$ \\
Sparse model: $\beta=0.5$ & $2.60 \times 10^{-4}$ & $3.11 \times 10^{-2}$ & $4.66 \times 10^{-2}$ & \\
\hline
\end{tabular}

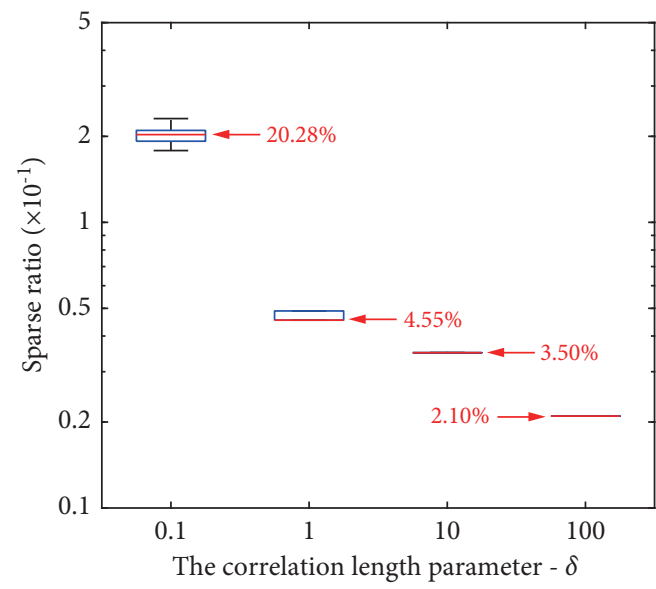

(a)

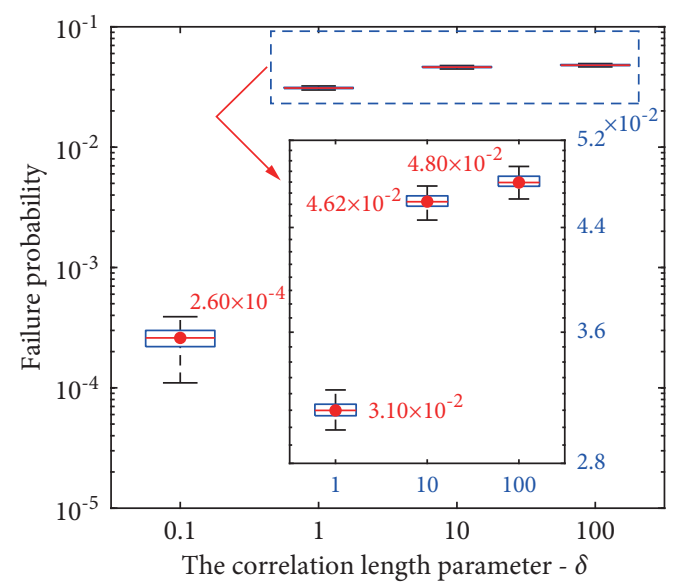

(b)

FIGURE 16: Results for the robustness analysis of the Gegenbauer polynomial-based surrogate model with parameter $\beta=0$. (a) The sparsity ratio. (b) Structural failure probability.

structural failure probability as presented in Figure 16(b). Note that a small variation result for the structural failure probability further verifies the robustness of the proposed
Gegenbauer polynomial-based regression method for reliability analysis of a structural model with dependent input uncertainties. 


\section{Conclusion}

Structural reliability analysis is typically evaluated based on a multivariate performance function that defines small failure probabilities. It is significant to develop a surrogate model to mimic the true performance function as the brutal-force MCS based on the realistic model might be computationally intensive. This paper presents utilizing the Gegenbauer polynomials to constitute the explanatory dictionary, whereas principle component functions are adaptively selected via the OMP-based sparse regression algorithm. Due to the regression bias introduced by utilizing random samples, an excluding procedure to detect spuriously component functions is proposed based on the $F$ statistics. Simulation results have shown that the Gegenbauer polynomial-based regression method can determine reliable estimation results for the investigated performance function. Small regression errors and the high sparsity of the surrogate model have demonstrated potential applications of the adaptive sparse regression algorithm for structural reliability analysis.

\section{Data Availability}

The simulation data within this submission are available based on the request.

\section{Conflicts of Interest}

The authors declare that they have no conflicts of interest.

\section{References}

[1] M. Rosenblatt, "Remarks on a multivariate transformation," The Annals of Mathematical Statistics, vol. 23, no. 3, pp. 470-472, 1952.

[2] P.-L. Liu and A. Der Kiureghian, "Multivariate distribution models with prescribed marginals and covariances," Probabilistic Engineering Mechanics, vol. 1, no. 2, pp. 105-112, 1986.

[3] S. Wang, "Reliability model of mechanical components with dependent failure modes," Mathematical Problems in Engineering, vol. 2013, Article ID 828407, 6 pages, 2013.

[4] R. E. Melchers, "Importance sampling in structural systems," Structural Safety, vol. 6, no. 1, pp. 3-10, 1989.

[5] J. Nie and B. R. Ellingwood, "Directional methods for structural reliability analysis," Structural Safety, vol. 22, no. 3, pp. 233-249, 2000.

[6] D. Meng, Y. Li, S.-P. Zhu, G. Lv, J. Correia, and A. de Jesus, "An enhanced reliability index method and its application in reliability-based collaborative design and optimization," Mathematical Problems in Engineering, vol. 2019, Article ID 4536906, 10 pages, 2019.

[7] G. I. Schuëller and H. J. Pradlwarter, "Benchmark study on reliability estimation in higher dimensions of structural systems-an overview," Structural Safety, vol. 29, no. 3, pp. 167-182, 2007.

[8] X. Zhang and M. D. Pandey, "Structural reliability analysis based on the concepts of entropy, fractional moment and dimensional reduction method," Structural Safety, vol. 43, pp. 28-40, 2013.
[9] H. P. Gavin and S. C. Yau, "High-order limit state functions in the response surface method for structural reliability analysis," Structural Safety, vol. 30, no. 2, pp. 162-179, 2008.

[10] X. Zhang and M. D. Pandey, "An effective approximation for variance-based global sensitivity analysis," Reliability Engineering \& System Safety, vol. 121, pp. 164-174, 2014.

[11] X. Zhang, L. Wang, and J. D. Sørensen, "AKOIS: an adaptive Kriging oriented importance sampling method for structural system reliability analysis," Structural Safety, vol. 82, p. 101876, 2020.

[12] X. Zhang, L. Wang, and J. D. Sørensen, "REIF: a novel activelearning function toward adaptive Kriging surrogate models for structural reliability analysis," Reliability Engineering \& System Safety, vol. 185, pp. 440-454, 2019.

[13] V. Cherkassky and Y. Ma, "Practical selection of SVM parameters and noise estimation for SVM regression," Neural Networks, vol. 17, no. 1, pp. 113-126, 2004.

[14] S. M. Clarke, M. Clarke, J. H. Griebsch, and T. W. Simpson, "Analysis of support vector regression for approximation of complex engineering analyses," Journal of Mechanical Design, vol. 127, no. 6, pp. 1077-1087, 2005.

[15] H. Fang and H. F. Mark, "Globle response approximation with radial basis functions," Engineering Optimization, vol. 38, no. 4, pp. 407-424, 2006.

[16] Y.-F. Zhang and Y.-L. Zhang, "Reliability sensitivity analysis method for mechanical components," Mathematical Problems in Engineering, vol. 2021, Article ID 7867003, 11 pages, 2021.

[17] O. P. Le Maîetre, M. T. Reagan, H. N. Najm, R. G. Ghanem, and O. M. Knio, "A stochastic projection method for fluid flow II.: random process," Journal of Computational Physics, vol. 181, no. 1, pp. 9-44, 2002.

[18] M. A. Tatang, W. Pan, R. G. Prinn, and G. J. Mcrae, “An efficient method for parametric uncertainty analysis of numerical geophysical models," Journal of Geophysical Research Atmospheres, vol. 102, no. 18, pp. 21925-21932, 1997.

[19] G. Blatman and B. Sudret, "An adaptive algorithm to build up sparse polynomial chaos expansions for stochastic finite element analysis," Probabilistic Engineering Mechanics, vol. 25, no. 2, pp. 183-197, 2010.

[20] V. Yaghoubi, S. Marelli, B. Sudret, and T. Abrahamsson, "Sparse polynomial chaos expansions of frequency response functions using stochastic frequency transformation," Probabilistic Engineering Mechanics, vol. 48, pp. 39-58, 2017.

[21] X. Zhang, M. D. Pandey, R. Yu, and Z. Wu, "HALK: a hybrid active-learning Kriging approach and its applications for structural reliability analysis," Engineering with Computers, 2021.

[22] J. Keiner, "Gegenbauer polynomials and semiseparable matrices," Electronic Transactions on Numerical Analysis, vol. 30, pp. 26-53, 2008.

[23] C. Ekanadham, D. Tranchina, and E. P. Simoncelli, "Recovery of sparse translation-invariant signals with continuous basis pursuit," IEEE Transactions on Signal Processing, vol. 59, no. 10, pp. 4735-4744, 2011.

[24] S. F. Cotter and B. D. Rao, "Sparse channel estimation via matching pursuit with application to equalization," IEEE Transactions on Communications, vol. 50, no. 3, pp. 374-377, 2002.

[25] J. A. Tropp and A. C. Gilbert, "Signal recovery from random measurements via orthogonal matching pursuit," IEEE Transactions on Information Theory, vol. 53, no. 12, pp. 4655-4666, 2007. 
[26] T. T. Cai and L. Wang, "Orthogonal matching pursuit for sparse signal recovery with noise," IEEE Transactions on Information Theory, vol. 57, no. 7, pp. 4680-4688, 2011.

[27] D. Needell and R. Vershynin, "Uniform uncertainty principle and signal recovery via regularized orthogonal matching pursuit," Foundations of Computational Mathematics, vol. 9, no. 3, pp. 317-334, 2009.

[28] D. L. Donoho, Y. Tsaig, I. Drori, and J.-L. Starck, "Sparse solution of underdetermined systems of linear equations by stagewise orthogonal matching pursuit," IEEE Transactions on Information Theory, vol. 58, no. 2, pp. 1094-1121, 2012.

[29] A. M. Hasofer and N. C. Lind, "Exact and invariant secondmoment code format," Journal of the Engineering Mechanics Division, vol. 100, no. 1, pp. 111-121, 1974.

[30] J. Keeiner, "Computing with expansions in Gegenbauer polynomials," SIAM Journal on Scientific Computing, vol. 31, no. 3, pp. 2151-2171, 2009.

[31] P. K. Suetin, Ultraspherical Polynomials, Encyclopedia of Mathematics, EMS Press, Berlin, Germany, 2001, https:// encyclopediaofmath.org/wiki/Ultraspherical_polynomials.

[32] E. M. Stein and G. Weiss, Introduction to Fourier Analysis on Euclidean Spaces, Princeton University Press, Princeton, NJ, USA, 1971.

[33] G. Schuëller, "Computational stochastic mechanics: recent advances," Computers \& Structures, vol. 79, no. 22-25, pp. 2225-2234, 2001.

[34] D. C. Montgomery, Design and Analysis of Experiments, John Wiley \& Sons, New York, NY, USA, 7th edition, 2009.

[35] H. Dai, H. Zhang, and W. Wang, "A new maximum entropybased importance sampling for reliability analysis," Structural Safety, vol. 63, pp. 71-80, 2016.

[36] R. G. Ghanem and P. D. Spanos, Stochastic Finite Elements: A Spectral Approach, Springer-Verlag, New York, NY, USA, 1991. 